\title{
BMJ Open Readiness of health facilities for the outpatient management of non- communicable diseases in a low- resource setting: an example from a facility-based cross-sectional survey in Tanzania
}

\author{
Deogratius Bintabara (D) ,' Dorothy Ngajilo ${ }^{2,3}$
}

To cite: Bintabara D, Ngajilo D. Readiness of health facilities for the outpatient management of non-communicable diseases in a low-resource setting: an example from a facility-based cross-sectional survey in Tanzania. BMJ Open 2020;10:e040908. doi:10.1136/ bmjopen-2020-040908

- Prepublication history and supplemental material for this paper is available online. To view these files, please visit the journal online (http://dx.doi. org/10.1136/bmjopen-2020040908).

Received 25 May 2020 Revised 30 August 2020 Accepted 23 September 2020

Check for updates

(C) Author(s) (or their employer(s)) 2020. Re-use permitted under CC BY-NC. No commercial re-use. See rights and permissions. Published by BMJ.

For numbered affiliations see end of article.

Correspondence to Dr Deogratius Bintabara; bintabaradeo@gmail.com

\section{ABSTRACT}

Objective This study assessed the readiness of health facilities to provide outpatient management of noncommunicable diseases using a nationally representative sample of health facilities from Tanzania as an example of a low-resource country.

Design Facility-based cross-sectional survey.

Setting This study analysed data collected from public and private-owned dispensaries/clinics, health centres and hospitals during the 2014-2015 Tanzania Service Provision Assessment survey.

Primary outcome measures Three outcome variables are included in this study, namely readiness of facilities to provide outpatient management for diabetes, hypertension and chronic respiratory diseases. These were composite variables measured based on availability of indicators identified in the WHO-Service Availability and Readiness Assessment manual. These indicators were grouped into three domains, viz staff training and guidelines, basic diagnostic equipment and basic medicines. Readiness was measured by assessing the presence of required indicators in each of these domains.

Results Out of 1188 health facilities assessed, $52.1 \%$, $64.8 \%$ and $60.9 \%$ reported providing services related to diabetes, hypertension and chronic respiratory diseases, respectively. A few facilities reported having treatment guidelines (33.2\%) or staff trained to provide noncommunicable disease services (10.4\%). The availability of basic diagnostic equipment and medicines for these diseases was significantly lower in public lower level facilities than in their private counterparts $(p<0.05)$. Facilities located in urban settings as well as higher level (health centre and hospitals) and publicly owned facilities were significantly associated with increased service readiness index for providing outpatient management of non-communicable diseases.

Conclusion A fair distribution of resources through the 'push' system of refresher training, treatment guidelines, medicines and diagnostic equipment from higher authorities or other agencies may be one way of strengthening the readiness of lower level and public facilities to cope with the increasing burden of non-
Strengths and limitations of this study

The main strength of this study is that it used a representative and comprehensive national data set from Tanzania, implying a high degree of accuracy in characterising the current situation of facility readiness to manage non-communicable diseases in low-resource countries.

- The provided estimates were adjusted and weighted to correct for non-response and disproportionate sampling.

- The use of cross-sectional survey meant that causality assumptions could not be inferred. Therefore, the present findings should be interpreted with caution.

communicable diseases in low-resource countries such as Tanzania.

\section{INTRODUCTION}

Nowadays, non-communicable diseases (NCDs) have been highlighted as a major challenge for sustainable development ${ }^{1} 2$ and an alarming public health challenge of global concern. ${ }^{4}$ These diseases, particularly cardiovascular diseases (CVD), diabetes mellitus (DM), cancers and chronic respiratory diseases (CRD), have been mentioned as the leading causes of disability and death globally, ${ }^{56}$ despite the reduced contribution of a $15 \%$ in premature mortality between 2000 and 2012. ${ }^{7}$ NCDs account for about 40 million adult deaths each year globally, of which $87 \%$ are premature deaths occurring in low and middle-income countries (LMICs). ${ }^{8}$ Over the next decade, the 54 LMICs in Africa are expected to become the world's largest contributors to NCD deaths. ${ }^{9}$ 
Despite communicable diseases (CDs) being the predominant health concern in Tanzania, the burden of NCDs has been increasing steadily ${ }^{10}$ with a recent report showing NCDs accounting for 409000 (33\%) of all deaths in 2016. ${ }^{11}$ This epidemiological transition has created a huge burden on the country's overstretched health system and its limited resources, ${ }^{12}{ }^{13}$ which was initially designed towards the management and treatment of CDs. ${ }^{14}$ Additionally, some reports have shown that the majority of primary healthcare facilities in LMICs are not prepared to manage NCDs. ${ }^{6}{ }^{14-16}$ In recognition of this challenge, the United Nations General Assembly (UNGA) met in 2011, and the participants emphasised the need to strengthen measures for the prevention and control of NCDs in LMICs.

The Tanzanian government through the Ministry of Health, Community Development, Gender Elderly and Children (MoHCDGEC) has complied with the UNGA by initiating and modifying its national NCD prevention and control strategy/policy, which is guided by the principle of ensuring access to and the availability of affordable quality NCD services throughout the country. ${ }^{17} 18$ Also, efforts have been made to increase the number of health facilities, healthcare workers, supply of diagnostic equipment and drugs. ${ }^{19-21}$ Currently, in each ward there is at least one dispensary and/or a health centre, each district has at least one hospital, while each region has at least one referral hospital. To ensure efficiency in management of patients, these facilities follow a referral chain with dispensaries at the bottom providing primary healthcare services mainly on outpatient basis, followed by health centres and then hospitals at the top of the referral chain. ${ }^{22}$ Despite these efforts, evidence shows an uneven distribution of these services according to location, managing authority (public vs private) and type of facility (dispensary, health centre or hospital).$^{2021}$ Moreover, the Tanzanian health sector continues to function under a shortage of funds from the government budget which is far below the Abuja declaration of $15 \% .{ }^{23}$ This makes it difficult for an already compromised health sector to incorporate NCD services at all health facilities.

The current study uses a nationwide representative sample of health facilities to describe the readiness of Tanzanian health facilities to provide NCD services by assessing the presence of treatment guidelines, trained staff members, basic medicine and diagnostic equipment for NCDs according to the type of facility and the managing authority. Furthermore, it assesses the factors associated with the level of facility readiness to provide NCD services. Assessing the readiness of health facilities for the outpatient management of NCDs is a crucial process in improving the quality of services provided, ${ }^{24}$ and a step towards strengthening prevention and control strategic frameworks. The obtained findings will be used to reinforce the previous efforts made in reducing mortality and disability mainly explained by a lack of access to basic medicine and diagnostic equipment for NCDs. ${ }^{25}$ The findings of this study may be applicable to other LMICs where information regarding the availability and readiness of health facilities for the outpatient management of NCDs is likely to be scarce.

\section{METHODS}

\section{Data source}

The current study used data from the 2014-2015 Tanzania Service Provision Assessment (TSPA) survey, which was designed to collect information about the availability and readiness of basic healthcare services among health facilities. The survey assessed the presence and function of components essential for the delivery of quality service for all aspects of healthcare including the management of NCDs. This survey was undertaken by Tanzania's National Bureau of Statistics and the Office of the Chief Government Statistician in collaboration with the MoHCDGEC-Mainland, and the Ministry of Health $(\mathrm{MoH})$-Zanzibar. It is the second facility-based survey conducted in Tanzania, following the one conducted in 2006. The survey was funded by the US Agency for International Development. ${ }^{26}$

\section{Study design, sample size and sampling techniques}

The 2014-2015 TSPA was a cross-sectional survey that assessed all formal sector health facilities in Tanzania. The health facilities were selected using a multistage cluster sampling technique from a sampling frame containing all health facilities in Tanzania. The sample size of 1200 was calculated according to facility type, managing authority and region to provide nationally representative results. The selection of desirable sampling units was achieved after excluding 12 facilities (7 which refused to participate, 4 which were closed on the interview days and 1 which could not be reached because of poor infrastructure). Therefore, a total of 1188 facilities (response rate of $99 \%$ ) were included in this analysis. However, to assess accurately the readiness of health facilities for the outpatient management of NCDs, we excluded 382 facilities which reported that they did not provide NCD services. Hence, the sample size of 619, 770 and 723 health facilities providing services for the management of DM, CVD (hypertension and/or mild symptoms of heart failure 'HT\&HF') and CRD, respectively, was involved in this analysis (figure 1).

\section{Data collection methods}

The 2014-2015 TSPA used four main types of questionnaires: a Facility Inventory Questionnaire; a Health Provider Interview Questionnaire, an Exit and Observation Protocols for antenatal care, family planning and sick child services. These questionnaires are adapted from the model questionnaires developed by the Demographic and Health Survey Program to include health issues relevant to Tanzania. Input was solicited from specialists and experts at the MoHCDGEC (Tanzania Mainland), the $\mathrm{MoH}$ (Zanzibar), development partners and other key stakeholders knowledgeable about health services in Tanzania. 


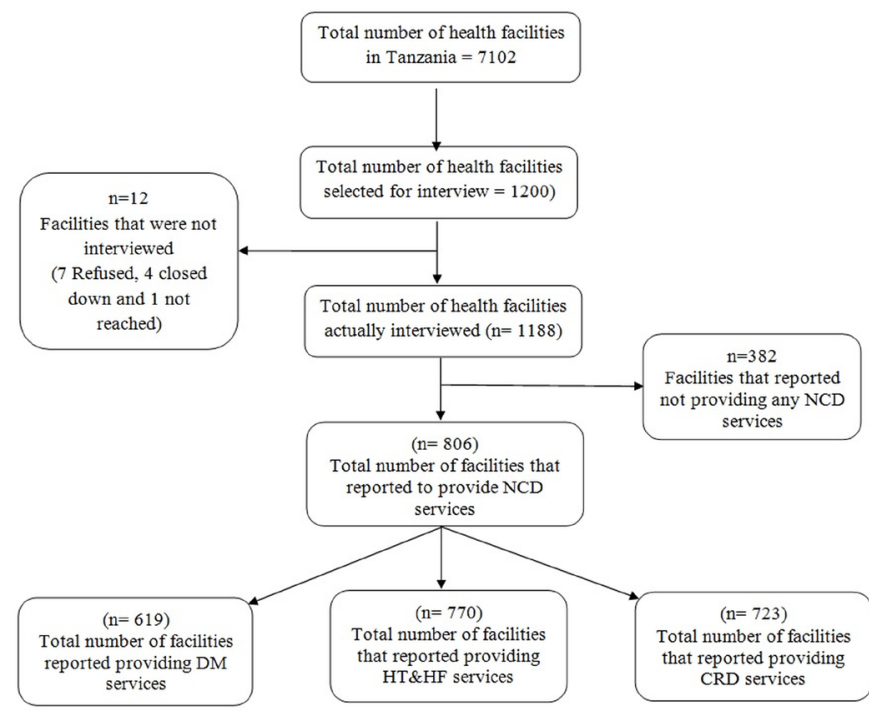

Figure 1 Selection procedure for the sampling units included in this analysis. Facility may report providing management for more than one disease. CRD, chronic respiratory disease; DM, diabetes mellitus; HT\&HF, hypertension and/or mild symptoms of heart failure; NCD, non-communicable disease.

However, this secondary analysis included variables from the Facility Inventory Questionnaire to answer our research questions. The questionnaire and variables used in the current study have been published elsewhere. ${ }^{27-29}$ The data for the 2014-2015 TSPA survey were collected between 20 October 2014 and 21 February 2015, and revisit of some facilities that were not covered previously was conducted from 2 to 13 March 2015. On average, data collection took 1 day for small facilities (dispensary, clinics and some health centres) and 2 or 3 days for large facilities (mostly hospitals).

\section{Measurement of variables}

\section{Outcome variables}

Three outcome variables are included in this study, namely the readiness of the facilities to provide outpatient management of DM, HT\&HF and CRD. These were composite variables created based on availability of NCD services-specific tracer indicators identified in the WHO-Service Availability and Readiness Assessment (WHO-SARA) reference manual. ${ }^{27}$ The selected indicators were also required to be available in the Tanzanian Standard Treatment Guideline (STG) and the National Essential Medicines List for Tanzania (NEMLIT).$^{30}$ Based on WHO-SARA reference manual, these indicators were grouped into three domains, viz (1) staff training and guidelines, (2) basic diagnostic equipment, and (3) basic medicines. Readiness was measured by assessing the presence of required indicators in each of those domains as described in detail in table 1. A composite readiness index for each outcome variable was calculated using a simple additive approach giving equal weight to each of the three domains and each of the indicators within the domains. Since the target total readiness index score for each variable outcome was $100 \%$, each of the three domains accounted for $33.3 \%$ of the total score. The weight of the individual indicators within the domains was calculated by dividing the weight of each domain (33.3\%) by the number of indicators in that domain. Finally, the NCD readiness index score for each facility was calculated as a sum of the percentages of the indicators found in that facility. ${ }^{31}{ }^{32}$ The details for the scoring of these outcome variables and the measurement process are summarised in online supplemental table 1.

\section{Explanatory variables}

The explanatory variables used in this study were facility location, facility type, managing authority, external supervision, quality assurance (ie, any quality assurance activities carried out during the past year), routine management meetings, external sources of revenue, user fee and health insurance. The selection of these variables was based on previous studies. ${ }^{31} 33$ The measurement process of these explanatory variables is summarised in more detail in online supplemental table 2.

\section{Statistical analysis}

The current study is based on the secondary analysis of data from the Facility Inventory file. Data cleaning was performed before analysis by calculating frequencies and sorting. As the facilities sampled were not evenly distributed and the response rate might be very different by regions or facility type, oversampling or undersampling in regions with fewer or more facilities respectively occurred before data collection. Therefore, before analysis, facility weight was applied by downweighting or upweighting in areas where there was oversampling or undersampling, respectively. This was done in order to restore the representativeness of the sample so that the total sample mimics the country's actual facility distribution.

Descriptive statistics were performed and the variables were summarised using tables and graphs. The availability of services (indicators) was compared according to type of facility (dispensary, health centre and hospital) and managing authority (public and private) using $\chi^{2}$ test. In addition, simple and multiple linear regressions were performed. All explanatory variables from simple linear regressions (unadjusted models) that showed an association with outcome variables at $\mathrm{p}<0.2$ were eligible for inclusion in multiple linear regression analyses (adjusted models). A t-test for each of the coefficients in multiple linear regression analyses was calculated and used to test for the association. A $p$ value $<0.05$ was considered to indicate statistical significance. STATA V.15 (StataCorp, College Texas) was used for all the analyses.

\section{Ethics statement}

This study was based on an analysis of existing public domain survey data sets that are freely available online with all identifier information detached. Informed consent was requested and obtained from the manager, the person in charge of the facility or the most senior 
Table 1 Domains and indicators for assessing the readiness outcome variables

Domain Indicator Data collection and description

1. Staff training Guidelines for the diagnosis and management of DM, Observed availability of National Standard Treatment and guidelines HT\&HF or CRD. Guidelines for NCDs which are provided in hard copy format at all facility levels in the outpatient department (OPD) or respective clinic for DM, HT\&HF or CRD.

Staff trained in the diagnosis and management of DM, HT\&HF or CRD.

Self-reported availability of at least one staff providing services who had completed refresher courses for the diagnosis and management of DM, HT\&HF or CRD within the last 2 years.

2. Basic DM: weight scale, height scale and glucometer.

diagnostic

equipment

HT\&HF: stethoscope and blood pressure (BP) apparatus.

CRD: stethoscope and peak flow-meter.

3. Basic DM: metformin, glibenclamide and insulin injection.
medicines

HT\&HF: thiazide diuretics, ACE inhibitors, beta blockers and furosemide.

CRD: inhaled bronchodilators, inhaled corticosteroid and prednisolone.
Observed availability of functioning weight scale, height scale and glucometer with test strips was never or only occasionally out of stock.

Observed availability of functioning digital or manual BP apparatus in the OPD or HT\&HF clinic.

Observed availability of functioning stethoscope and peak flow-meter in the OPD or CRD clinic.

Observed availability of a first-line regimen that had not expired; metformin (for obese patients), glibenclamide (for non-obese patients) and insulin injection for type $1 \mathrm{DM}$.

Observed availability of at least one type of thiazide diuretics as a first-line regimen for hypertension and at least one regimen for the management of heart failure (ACE inhibitor+beta blocker+furosemide).

Observed availability of inhaled bronchodilators

(salbutamol),

inhaled corticosteroid (beclomethasone) or prednisolone tablets.

Since CVD comprised several diseases, this study opted to include HT and mild HF because they can be managed as OPD cases, enabling all types of facilities to be included in the study.

CRD, chronic respiratory disease; CVD, cardiovascular disease; DM, diabetes mellitus; HT\&HF, hypertension and/or mild symptoms of heart failure; NCD, non-communicable disease.

healthcare worker responsible for client services present at the facility. The respondents were adequately informed regarding all relevant aspects of the study, including its aim and interview procedures. Respondents who accepted for their facilities to participate in the study provided signed written informed consent.

\section{Patient and public involvement}

Patients and the public were not involved in the analysis of this study.

\section{RESULTS}

\section{Background characteristics of surveyed health facilities}

Out of 1188 health facilities assessed in the TSPA survey, majority $(864,72.7 \%)$ were located in rural settings. Most of the facilities $(857,72.2 \%)$ were public facilities operated by government authorities and many (1013, $85.3 \%$ ) were dispensaries or clinics. The majority (697, $58.7 \%$ ) reported receiving funds from the government as an external source of revenue, while only 499 (42.0\%) reported accepting at least one type of health insurance scheme. Among all the health facilities, 619 (52.1\%), 770
$(64.8 \%)$ and 723 (60.8) reported providing management or service related to DM, HT\&HF and CRD, respectively. Few facilities were observed to have at least one treatment guideline related to NCDs $(394,33.2 \%)$ or at least one health provider who had received refresher course related to NCDs $(123,10.4 \%)$ (table 2$)$.

\section{Availability of trained staff and guidelines}

The availability of guidelines for the management of DM, HT\&HF and CRD was found to differ significantly according to the type of facility, with lower availability at dispensary level than hospitals $(\mathrm{p}<0.05)$. No difference in the availability of guidelines according to the managing authority was seen except at health centres where more public than private sector facilities reported having treatment guidelines for NCDs $(p<0.05)$. The proportion of facilities with at least one staff who had received refresher training on outpatient management of NCDs was low across all types of facilities and managing authorities. Furthermore, the availability of these trained staff was significantly higher in hospitals and health centres than dispensaries $(\mathrm{p}<0.05)$ as well as in public than private 
Table 2 Per cent distribution of surveyed facilities according to background characteristics, TSPA 2014-2015 $(n=1188)$

\begin{tabular}{|c|c|c|}
\hline Variable & n (weighted) & $\%$ \\
\hline \multicolumn{3}{|l|}{ Facility location } \\
\hline Urban & 324 & 27.3 \\
\hline Rural & 864 & 72.7 \\
\hline \multicolumn{3}{|l|}{ Managing authority } \\
\hline Public & 857 & 72.2 \\
\hline Private & 331 & 27.8 \\
\hline \multicolumn{3}{|l|}{ Facility type } \\
\hline Clinic and dispensary & 1013 & 85.3 \\
\hline Health centre & 129 & 10.8 \\
\hline Hospital & 46 & 3.9 \\
\hline \multicolumn{3}{|l|}{ External source of revenue } \\
\hline Government & 697 & 58.7 \\
\hline Other than government & 379 & 31.9 \\
\hline None & 112 & 9.4 \\
\hline \multicolumn{3}{|l|}{ Health insurance* } \\
\hline Not accepted & 689 & 58.0 \\
\hline Accepted & 499 & 42.0 \\
\hline \multicolumn{3}{|c|}{ Type of NCD services available† } \\
\hline Diabetes & 619 & 52.1 \\
\hline CVD & 770 & 64.8 \\
\hline CRD & 723 & 60.8 \\
\hline \multicolumn{3}{|l|}{ Guidelines for NCD } \\
\hline No available & 794 & 66.8 \\
\hline Available & 394 & 33.2 \\
\hline \multicolumn{3}{|l|}{ Staff trained for NCD } \\
\hline No available & 1065 & 89.6 \\
\hline Available & 123 & 10.4 \\
\hline
\end{tabular}

${ }^{*}$ Any kind of health insurance (government or private). †n and \% do not add up to 1188 and $100 \%$, respectively, because multiple responses were possible.

CRD, chronic respiratory disease; CVD, cardiovascular disease; NCD, non-communicable disease; TSPA, Tanzania Service Provision Assessment.

facilities $(\mathrm{p}<0.05)$, except at the dispensary level where private facilities performed better $(\mathrm{p}<0.05)$ (figure 2$)$.

\section{Availability of basic diagnostic equipment}

Basic diagnostic equipment for DM

Regardless of the facility type and managing authority, the availability of a functioning adult weight scale was consistently high across all facilities at $85.6 \%, 96.2 \%, 79.5 \%$, $84.5 \%, 71.2 \%$ and $87.1 \%$ for public hospitals, private hospitals, public health centres, private health centres, public dispensaries and private dispensaries, respectively. The availability of a functioning height scale ranged from $37.0 \%$ to $71.0 \%$, exhibiting significant differences according to type of facility and managing authority

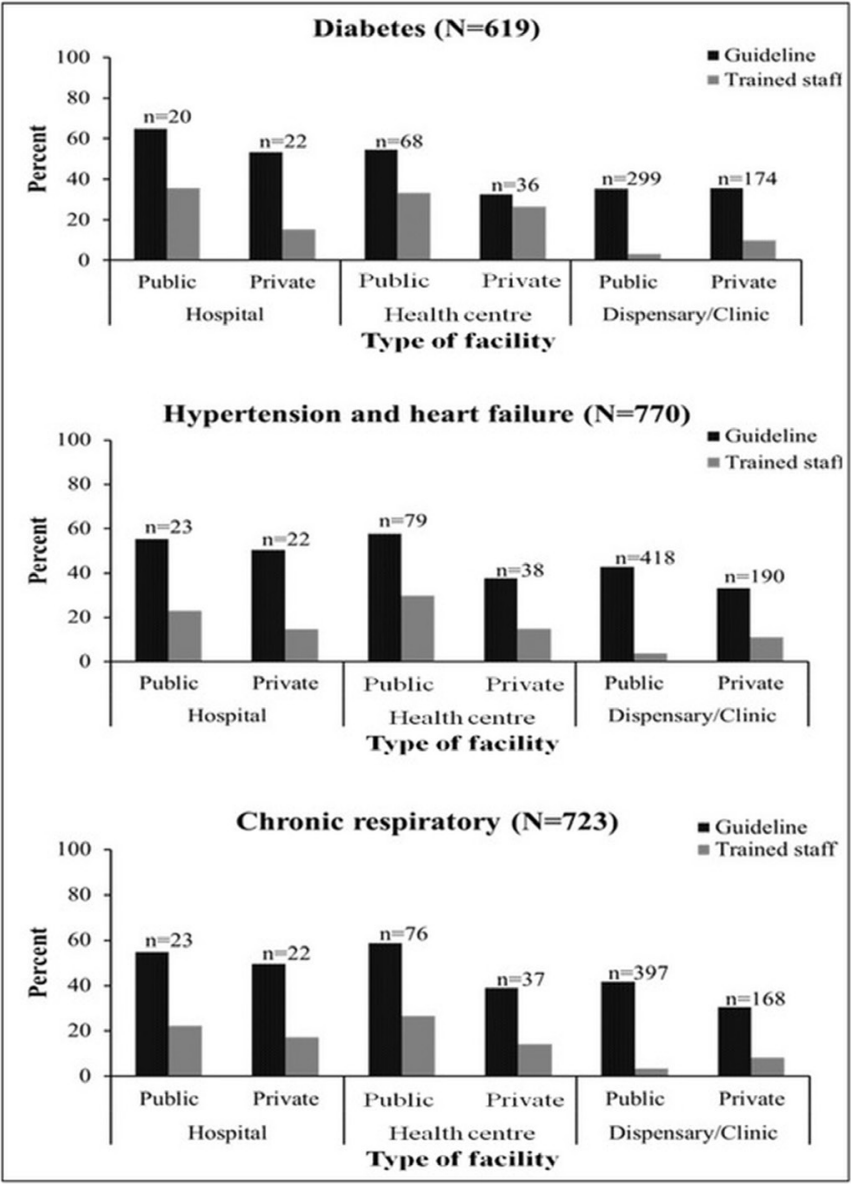

Figure 2 Availability of guidelines and at least one staff member trained to ensure the quality of outpatient management of non-communicable disease (NCD) according to type of facility and managing authority.

$(p<0.05)$. Significantly lower proportion of functioning glucometers with testing strips was found in public facilities as compared with private facilities $(\mathrm{p}<0.05)$. Overall, availability of basic diagnostic equipment for DM was higher in private $(63.1 \%, 43.7 \%$ and $27.2 \%)$ than public facilities $(44.2 \%, 25.9 \%$ and $2.8 \%)$ for hospitals, health centres and dispensaries, respectively $(\mathrm{p}<0.05)$.

Basic diagnostic equipment for HT\&HF

The availability of a functioning blood pressure (BP) apparatus (manual or digital) and a stethoscope was consistently high $\quad(85.1 \%-97.7 \%$ and $\quad 91.0 \%-98.0 \%$, respectively). These observed availabilities did not differ significantly according to the type of facility and managing authority $(\mathrm{p}>0.05)$.

\section{Basic diagnostic equipment for CRD}

The availability of a peak flow-meter was consistently low $(0.0 \%-28.6 \%)$ in all types of health facilities and managing authorities. With exception of dispensaries, however, significant differences in reporting for this item were observed according to the managing authority, with availability being higher in private $(17.8 \%, 28.6 \%$ and $1.9 \%)$ than public facilities $(7.4 \%, 4.6 \%$ and $0.0 \%)$ for 


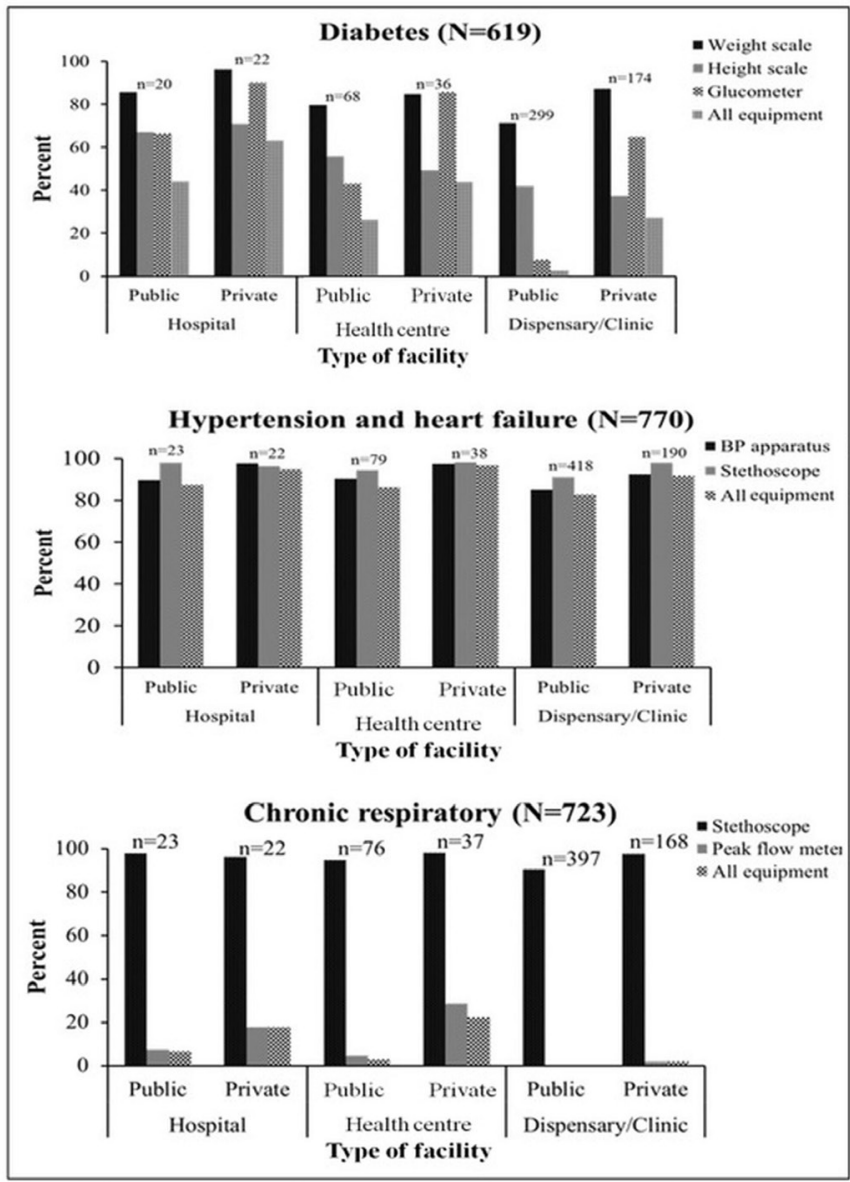

Figure 3 Availability of basic diagnostic equipment necessary to ensure the quality of outpatient management for non-communicable disease (NCD) according to type of facility and managing authority. BP, blood pressure.

hospitals, health centres and dispensaries, respectively $(\mathrm{p}<0.05)$ (figure 3).

\section{Availability of basic medicines}

Basic medicines for DM

The overall availability of metformin, glibenclamide and injectable insulin was consistently high for both public and private hospitals, compared with health centres and dispensaries. With the exception of hospitals, significant differences in availability of these three antidiabetic medicines were observed according to managing authority, with private health centres reporting higher availability $(72.9 \%$, $59.6 \%$ and $31.4 \%)$ than public health centres $(20.2 \%, 18.3 \%$ and $8.3 \%$ ) for metformin, glibenclamide and injectable insulin, respectively $(\mathrm{p}<0.01)$. Similarly, private dispensaries reported higher availability $(42.0 \%, 34.1 \%$ and $6.1 \%)$ than public dispensaries $(5.2 \%, 2.9 \%$ and $0.1 \%)$ for metformin, glibenclamide and injectable insulin, respectively $(\mathrm{p}<0.01)$. Of these three basic medicines, injectable insulin was found to be rarely available at lower level health facilities, particularly in public dispensaries owned by government.

\section{Basic medicines for HT\&HF}

The availability of at least one type of thiazide diuretics as a first-line regimen for hypertension was consistently low $(0.0 \%-28.5 \%)$ for all facilities. However, significant differences in the availability of thiazide diuretics according to type of facility and managing authority were found with private facilities reporting higher availability $(28.5 \%, 16.3 \%$ and $11.1 \%)$ than public facilities $(20.6 \%$, $3.1 \%$ and $0.0 \%$ ) for public hospitals, health centres and dispensaries, respectively $(\mathrm{p}<0.01)$. Similarly, significant differences in the overall availability of at least one basic regimen for heart failure were observed where private facilities reported a higher availability $(79.3 \%, 60.6 \%$ and $20.6 \%)$ than public facilities $(49.5 \% .7 .3 \%$ and $0.5 \%)$ at hospitals, health centres and dispensaries, respectively $(\mathrm{p}<0.01)$.

\section{Basic medicines for CRD}

The availability of inhaled salbutamol was higher at hospitals $(48.4 \%-71.8 \%)$ than at health centres $(13.8 \%-41.2 \%)$ and dispensaries $(6.8 \%-32.3 \%)(\mathrm{p}<0.05)$. However, the availability of inhaled beclomethasone was consistently low $(0.0 \%-16.4 \%)$ at all levels of health facilities. Availability varied according to the managing authority, with no public facilities reporting the availability of this item. The availability of prednisolone was higher at private facilities $(93.9 \%, 87.2 \%$ and $68.1 \%)$ than public facilities $(69.5 \%, 40.6 \%$ and $13.6 \%)$ for hospitals, health centres and dispensaries, respectively $(\mathrm{p}<0.05)$ (figure 4$)$.

\section{Readiness of facility to provide outpatient management of NCDs}

Table 3 presents the means of the composite readiness scores with $95 \%$ CIs for each outcome variable according to level of facility. While the overall mean readiness scores for all three outcomes were relatively low, hospitals performed better than other facility levels. Furthermore, the overall mean readiness of facilities to provide HT\&HF services was higher $(46.37 \%)$ than that for DM $(32.49 \%)$ and for CRD (30.83\%).

\section{Factors associated with readiness of facilities to provide outpatient management of NCDs}

Table 4 presents the results of unadjusted and adjusted linear regression analyses evaluating the association between service readiness indices and a range of explanatory variables. The results of adjusted analyses (models $1 \mathrm{~b}, 2 \mathrm{~b}$ and $3 \mathrm{~b}$ ) show that the service readiness indices of facilities to provide DM, HT\&HF and CRD services were significantly higher in urban than rural facilities; health centres and hospitals than dispensaries; privately than publicly owned facilities, and in facilities performing routine management meetings than facilities without such meetings, holding constant all other variables in the models.

\section{DISCUSSION}

This study describes the readiness of health facilities for the outpatient management of NCDs such as DM, HT\&HF and CRD in Tanzania, a low-resource country, 


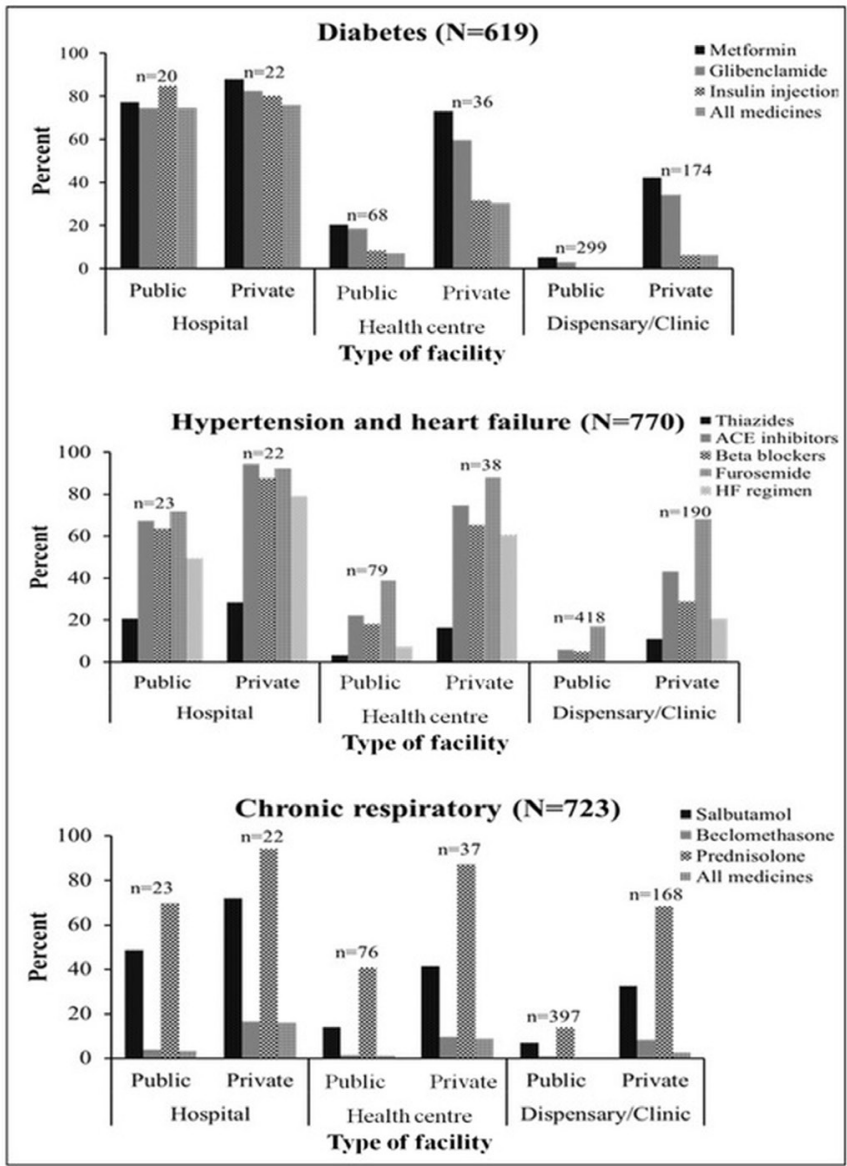

Figure 4 Availability of basic medicines necessary to ensure the quality of outpatient management of non-communicable disease (NCD) according to type of facilities and managing authorities. HF, heart failure.

by assessing the presence of treatment guidelines, trained staff members, basic medicines and diagnostic equipment for NCDs according to the type of facility and the managing authority. Overall, the study found variations in readiness to offer NCD services according to the type of facility and managing authority. Higher level facilities (hospitals) generally had a higher level of readiness than lower level facilities (health centres and dispensaries). Similarly, privately owned facilities, and facilities that reported to perform routine management meetings had higher service readiness index compared with their counterparts.

When looking at individual preidentified domains (staff training and guidelines, basic diagnostic equipment and basic medicines), the current study found significant differences in the availability of guidelines for the treatment of DM, HT\&HF and CRD according to the type of facility, with dispensaries having a lower availability than hospitals. This finding is in agreement with that of previous studies conducted in north-western Tanzania and Uganda. ${ }^{15} 28$ This low availability of treatment guidelines at a lower level of healthcare is a counter to evidence that the presence of well-established guidelines can be effective for the prevention and management of NCD. ${ }^{35}{ }^{36}$ Also, the study found that a low proportion of staff members had received a refresher course for the management of NCDs across all levels of health facilities. Similar findings have been reported in Cambodia ${ }^{37}$ and Vietnam. ${ }^{38}$ The similarity of these findings might be due to the fact that these countries are categorised as LMICs; therefore, they are more likely to face similar financial challenges resulting in the allocation of relatively small budget for the health sector, thereby compromising the prevention and management of NCDs.

Evidence shows that the availability of basic diagnostic equipment has an impact on early detection and clinical management of NCDs. ${ }^{39}$ However, the current study found significant amounts of variation and disproportion in the availability of some diagnostic equipment for NCDs. Regarding the diagnosis and screening of risk factors for diabetes, the availability of weight scales was consistently high among all levels of health facilities and managing authorities. A similar finding was obtained in a study conducted in Cambodia. ${ }^{37}$ The high reported availability of this necessary component for calculating body mass index (BMI) in both studies may be explained by the fact that weight scales are also used for other purposes such as drug dosing, anaesthesia, radiation dosage and clinical monitoring. The utility of weight scales may explain why the availability of weight scales was not proportional to the availability of height scales, which are another necessary component for calculating BMI. The current study found a relatively low and significant difference in the

Table 3 Readiness score to provide outpatient management of NCDs, TSPA 2014-2015

\begin{tabular}{|c|c|c|c|}
\hline & DM & HT\&HF & CRD \\
\hline & $\begin{array}{l}\text { Percentage mean score } \\
(95 \% \mathrm{Cl})\end{array}$ & $\begin{array}{l}\text { Percentage mean score } \\
(95 \% \mathrm{Cl})\end{array}$ & $\begin{array}{l}\text { Percentage mean score } \\
(95 \% \mathrm{Cl})\end{array}$ \\
\hline Health centre & $43.30(41.03$ to $45 / 57)$ & 55.26 (53.48 to 57.04$)$ & 39.14 (37.51 to 40.77$)$ \\
\hline Hospital & 67.56 (65.46 to 69.63$)$ & 65.58 (61.40 to 69.77$)$ & 47.02 (44.04 to 49.99$)$ \\
\hline
\end{tabular}

CRD, chronic respiratory disease; DM, diabetes mellitus; HT\&HF, hypertension and/or mild symptoms of heart failure; NCD, noncommunicable disease; TSPA, Tanzania Service Provision Assessment. 
Table 4 Factors associated with readiness of facilities to provide outpatient management of NCDs in unadjusted and adjusted linear regression models, TSPA 2014-2015

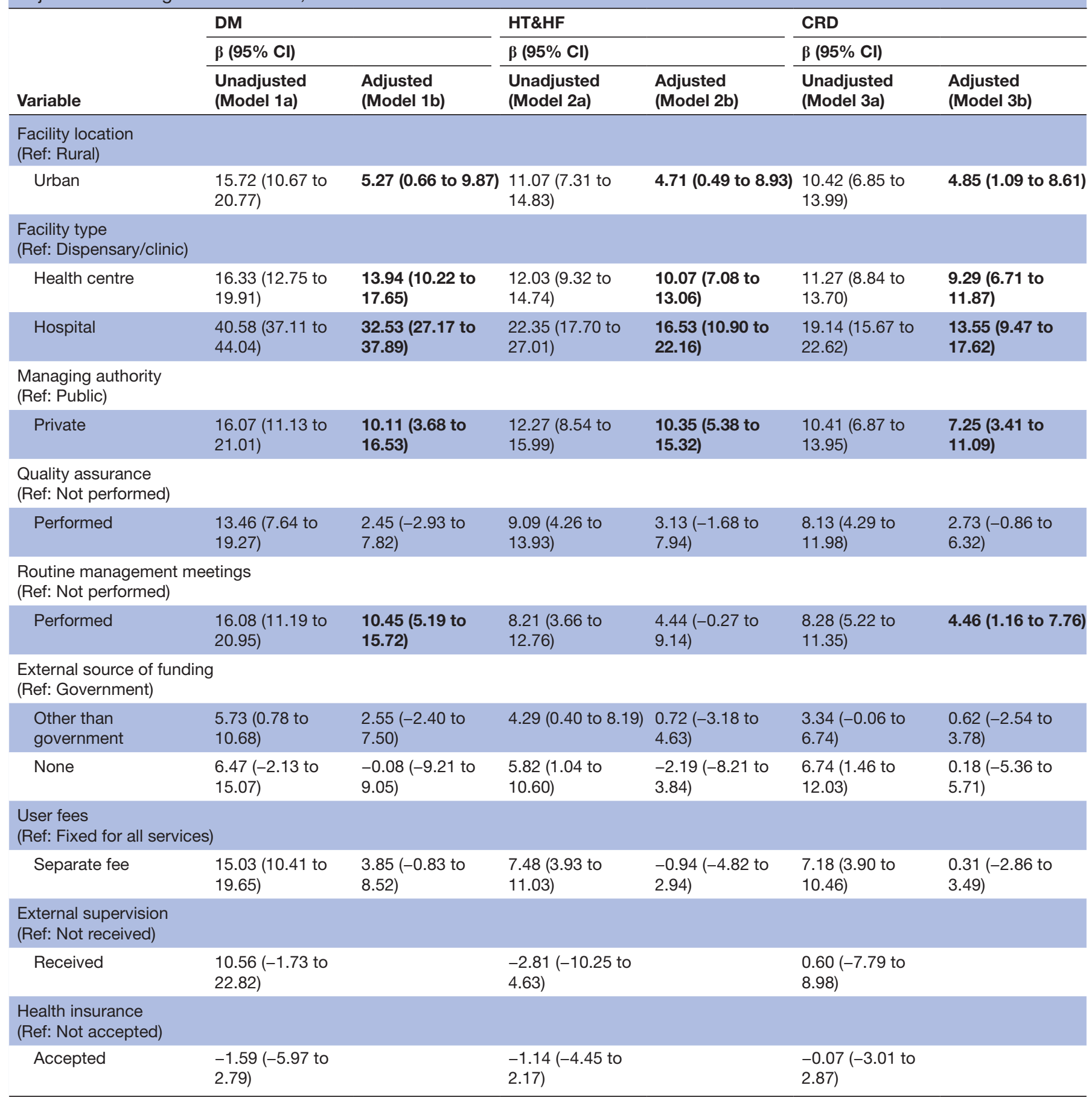

Bold values indicates the variable is statistically significant at $P$-value less than 0.05 .

$\beta$, coefficient; CRD, chronic respiratory disease; DM, diabetes mellitus; HT\&HF, hypertension and/or mild symptoms of heart failure; NCD, non-communicable disease; TSPA, Tanzania Service Provision Assessment.

availability of height scales according to the type of facility and managing authority. This result can be explained by the fact that height scales are more limited in their use compared with weight scales, hence their availability in adult outpatient department depends on specific purpose, such as calculating BMI. Furthermore, this study found significant differences in the availability of glucometers and testing strips according to the type of facility and managing authority. Similar findings were observed in the studies conducted in Uganda where a higher proportion of glucometers were found in hospitals than in health centres and dispensaries, ${ }^{28}$ and in Ethiopia where these items were more available in private facilities than in public facilities. ${ }^{40}$ The present study found that a relative 
high proportion of facilities reported the availability of $\mathrm{BP}$ apparatus and stethoscope as basic diagnostic equipment for the management of HT\&HF. Also, the study observed no significant difference in the availability of these items according to the type of facility or managing authority. Similar findings have also been reported by previous studies conducted in other African countries such as Uganda, Ethiopia and Nigeria. ${ }^{28} 4041$ This finding may be due to the fact that the possession of a stethoscope or BP apparatus is considered a mark of identity and prestige for clinicians/prescribers. ${ }^{42}$ However, higher availability might also be due to the increasing numbers of patients with elevated BP in these countries. ${ }^{43-45}$ Peak flow-meters are commonly used in the diagnosis and follow-up of CRD in many LMICs instead of more accurate devices such as spirometers, because other devices are more expensive and require regular technical maintenance. ${ }^{46}$ Therefore, the presence of peak flow-meters was used to denote the availability of basic diagnostic equipment for CRD. The findings from this study show that a low proportion of health facilities reported the availability of peak flowmeters with private facilities reporting higher availability than public facilities. This low proportion observed in the current study may be attributed to a low level of knowledge among healthcare workers on the usefulness of a peak flow-meter in the diagnosis and monitoring of patients with CRD as reported in other studies. ${ }^{47} 48$

Access to effective treatment is a crucial step in reducing the burden of $\mathrm{NCDs}^{49}$; however, services remain limited according to the type of facility and managing authority in many LMICs. ${ }^{5051}$ Similar to findings from other studies ${ }^{5152}$ the current study observed a varied availability of basic medicines for NCDs according to the type of facility and managing authority. Overall, the availability of metformin, glibenclamide and injectable insulin reported in this study was consistently higher at higher level facilities (hospitals) than at lower level facilities (health centres and dispensaries). Furthermore, private facilities reported higher availability of these antidiabetes medications than public facilities. Similar findings have been reported by previous studies conducted in Tanzania ${ }^{345253}$ and elsewhere. ${ }^{28} 5455$ Additionally, the study found a low proportion of health facilities reporting the availability of at least one type of thiazide diuretics as a first-line regimen for hypertension despite this drug being an inexpensive antihypertensive agent. ${ }^{56}$ Similarly, this study found low availability of at least one basic regimen (ACE inhibitor+beta blocker+furosemide) for heart failure management. However, the availability of these medicines was high at hospitals than health centres and dispensaries and at private compared with public facilities. These findings are in agreement with other previous studies conducted in LMICs such as Uganda, ${ }^{15}$ Zambia, ${ }^{16}$ Nigeria $^{41}$ and Cameroon. ${ }^{57}$ Salbutamol and beclomethasone inhalers are among the basic recommended CRD medicines included in the package of essential NCD interventions for primary healthcare. ${ }^{58}$ However, several previous studies have reported poor availability of these medicines particularly in public facilities in LMICs. ${ }^{55960}$ In the present study, the overall reported availability of beclomethasone was lower than that of salbutamol. These findings are similar to those of previous studies ${ }^{55} 59-62$ which found a significant variation in the availability of these medicines according to the managing authority, with private facilities having higher availability than public facilities.

The observed variations in the availability of services for outpatient management of NCDs according to the type of facility and managing authority found in this study may be due to several reasons, such as the complexity of the procurement process for basic medicines and diagnostic equipment for public health facilities. With the exception of hospitals that place their orders centrally at the Medical Stores Department (MSD), an agency responsible for the procurement and distribution of medical supplies to government health facilities, the remaining facilities follow a complex procurement process involving a district medical officer (DMO), who is identified as a key person in the pharmaceutical supply chain for these facilities. Therefore, after a facility determines its needs, it places an order on a quarterly basis using an indent/ integrated logistics system ('pull' system) to the MSD, with which each facility has its own account funded by the government. However, these orders must be reviewed and approved by the DMO. The MSD packs the supplies and delivers them to the DMO, who then distributes them to the health facilities. ${ }^{1963}$ Despite the fact that each facility generates an income (from cost sharing and health insurance sources) and receives a basket fund (from external agencies), the use of these funds to purchase medical supplies from private pharmacies requires permission and approval from the DMO. This complex and bureaucratic procurement process may cause delays and shortages of medicines and diagnostic equipment in health facilities. Another reason may be shortage at the MSD, which can run out of stock since it purchases products from international suppliers strictly on an annual ordering basis. Sometimes deliveries from suppliers take longer than expected, and the MSD may temporarily run out of stock. ${ }^{64}$ Furthermore, inadequate budget allocations to health facilities, delays in the distribution of allocated funds, inaccurate forecasting at the facility and national level, insufficient ordering of NCD medicines and equipment as well as theft of these items within health facilities can also lead to shortages. It is also important to highlight the fact that lower level facilities are limited to provide adequate NCD services because of the restrictions imposed by the NEMLIT which limits some of the basic NCD medicines only to higher level facilities. We recommend that the NEMLIT should be revised in order to ensure that essential NCD medicines restricted to higher level facilities are made available at lower level facilities. This will likely to improve the availability of NCD services at lower level facilities.

The variations in availability of services based on these domains have impact on the readiness of facilities to provide outpatient management of NCDs. Therefore, 
with these variations, we aimed to identify which factors might be associated with high readiness of facilities to provide outpatient management of NCDs. While Tanzanian health policies and guidelines require health facilities at all levels to provide basic management for NCDs, this study found facilities located in urban setting as well as higher level and privately owned facilities were more likely to have higher readiness index for the outpatient management of NCDs. The poor readiness observed in the lower level facilities (dispensaries/clinics) may be due to imprecise formula on how to distribute funds in these facilities which may contribute to inadequacies and inequities in the distribution of medical supplies and health resources. ${ }^{63}$

The findings of this study provide valuable policy implications to improve NCD services at all facilities regardless of level or managing authority. This includes enhanced country-wide commitment to offer NCD services in all facilities as stipulated in the existing policies and guidelines. There is also a need to develop strategies that will facilitate attainment of better policy outcomes taking into account local contextual factors such as political will, resource allocation as well as availability of data for monitoring policy impacts. Policies for fund allocation and procurement should be revised in order to strengthen lower level facilities particularly publicly owned and those located in the rural areas serving majority of the Tanzanian population. This may be the initial step for the country to fight against the rising burden of the major NCDs through outpatient care. Also, efforts should be made to meet the voluntary target set by Global Action Plan on NCDs of an $80 \%$ availability of affordable basic technologies and medicines required to treat major NCDs in both public and private facilities. ${ }^{9}$ Through this, Tanzania can be in a better position to achieving a target number 3 of Sustainable Development Goals by 2030, aimed to reduce by one-third premature mortality from NCDs. ${ }^{2}$

The main strength of this study is that it used a representative and comprehensive national data set from a low-resource country, implying a high degree of accuracy in characterising the current situation in low-resource countries. The outcome variables were created based on indicators identified from the WHO-SARA manual, which were required also to be available in the STG and NEMLIT to reflect the clinical reality of the study setting. Furthermore, the provided estimates were adjusted and weighted to correct for non-response and disproportionate sampling. Nevertheless, the cross-sectional nature of the study is a limitation, and the study failed to assess the trends in availability of items, which might have affected the readiness of some facilities. Therefore, the present findings should be interpreted with caution. As secondary data were used in this analysis, we missed some important information such as the reasons for some facilities not providing NCD services. Moreover, since we do not have information on the facilities that refused to participate in the study, we were unable to show if they were systematically different from those that agreed to participate. Thus, we cannot rule out potential nonresponse bias.

In summary, the present study provided empirical evidence of the variation in readiness for outpatient management of NCDs according to the type of facility and the managing authority in Tanzania. The present results highlighted a gap in the availability of treatment guidelines and trained staff members, basic medicines and diagnostic equipment, with relatively poor availability reported by public and lower level health facilities, resulting in poor readiness to offer NCD services. A comprehensive linkage of the procurement process should be established between lower level health facilities and the MSD, so that these facilities can have full autonomy to order and receive medicines and other medical equipment direct from the MSD without DMO approval. This may help overcome unnecessary delays or out of stock of NCD items arising from complexities of the current ordering process. Also, fair distribution through 'push' system of refresher courses, treatment guidelines, medicines and diagnostic equipment from higher authorities or other agencies is recommended as one of the interventions to address the increasing burden of NCDs in low-resource countries such as Tanzania.

\section{Author affiliations}

${ }^{1}$ Department of Community Medicine, School of Medicine, The University of Dodoma, Dodoma, Tanzania

${ }^{2}$ Ministry of Health, Community Development, Gender Elderly and Children, Dodoma, Tanzania

${ }^{3}$ Occupational Medicine Division/Centre for Environmental and Occupational Health Research, School of Public Health and Family Medicine, University of Cape Town, Cape Town, South Africa

Acknowledgements We would like to acknowledge the Demographic and Health Survey Program for giving us permission and access to the Tanzania Service Provision Assessment 2014-2015 data set.

Contributors DB originated the design of the study, performed statistical analysis and interpretation of data. DB and DN drafted, critically revised and approved the final manuscript for submission.

Funding The authors have not declared a specific grant for this research from any funding agency in the public, commercial or not-for-profit sectors.

Competing interests None declared.

Patient consent for publication Not required.

Ethics approval The survey was approved by the Ethics Committee of the ICF Macro at Calverton in the USA and by the National Institute of Medical Research Ethics Committee in Tanzania.

Provenance and peer review Not commissioned; externally peer reviewed.

Data availability statement Data are available in a public, open access repository. The data sets generated during the current study are available from the Demographic and Health Survey Program repository: http://dhsprogram.com/data/ available-datasets.cfm.

Supplemental material This content has been supplied by the author(s). It has not been vetted by BMJ Publishing Group Limited (BMJ) and may not have been peer-reviewed. Any opinions or recommendations discussed are solely those of the author(s) and are not endorsed by BMJ. BMJ disclaims all liability and responsibility arising from any reliance placed on the content. Where the content includes any translated material, BMJ does not warrant the accuracy and reliability of the translations (including but not limited to local regulations, clinical guidelines, terminology, drug names and drug dosages), and is not responsible for any error and/or omissions arising from translation and adaptation or otherwise. 
Open access This is an open access article distributed in accordance with the Creative Commons Attribution Non Commercial (CC BY-NC 4.0) license, which permits others to distribute, remix, adapt, build upon this work non-commercially, and license their derivative works on different terms, provided the original work is properly cited, appropriate credit is given, any changes made indicated, and the use is non-commercial. See: http://creativecommons.org/licenses/by-nc/4.0/.

ORCID iD

Deogratius Bintabara http://orcid.org/0000-0001-7877-870X

\section{REFERENCES}

1 Schmidt H, Barnhill A. Equity and noncommunicable disease reduction under the sustainable development goals. PLoS Med 2015;12:e1001872.

2 United Nations (UN). The sustainable development goals report 2016, 2016. Available: https://www.un-ilibrary.org/economic-andsocial-development/the-sustainable-development-goals-report2016_3405d09f-en https://www.who.int/goe/policies/countries/tza_ ehealth.pdf [Accessed 30 Aug 2020].

3 World Health Organization. Global status report on noncommunicable diseases, 2010. ISBN: 9789241564229.

4 Bloom DE, Cafiero ET, Jané-Llopis E, et al. The global economic burden of non-communicable diseases. World Econ Forum 2011.

5 World Health Organization. Global health risks: mortality and burden of disease attributable to selected major risks. Bull World Health Organ 2009.

6 Naik R, Kaneda T. Noncommunicable diseases in Africa: youth are the key to curbing the epidemic and achieveing sustainable development, 2015. Available: http://www.prb.org/pdf15/ncds-africapolicybrief.pdf [Accessed 30 Aug 2020].

7 World Health Organization. Health in 2015: from MDGs, millennium development goals to SDGs, sustainable development goals, 2015 Available: https://www.who.int/publications/i/item/9789241565110

8 World Health Organization. Noncommunicable diseases: fact sheet (updated January 2015), 2015. Available: http://www.who.int/ mediacentre/factsheets/fs355/en/ [Accessed 30 Aug 2020].

9 World Health Organization. Global action plan for the prevention and control of noncommunicable diseases 2013-2020. Available: https:// apps.who.int/iris/bitstream/handle/10665/94384/9789241506236_ eng.pdf;jsessionid=15BC796C3931494953006DFA64CDE7C9? sequence $=1$ [Accessed 30 Aug 2020].

10 Mayige M, Kagaruki G, Ramaiya K, et al. Non communicable diseases in Tanzania: a call for urgent action. Tanzan J Health Res 2011;13:378-86.

11 World Health Organization (WHO). Noncommunicable diseases country profiles 2018,2018 . Available: https://www.who.int/nmh/ publications/ncd-profiles-2018/en/

12 Ministry of Health \& Social Welfare (MoHSW). Tanzania national eHealth strategy 2012-2018; 2013 [Accessed 30 Aug 2020].

13 Miranda JJ, Kinra S, Casas JP, et al. Non-Communicable diseases in low- and middle-income countries: context, determinants and health policy. Trop Med Int Heal 2008;13:1225-34.

14 Beaglehole R, Epping-Jordan J, Patel V, et al. Improving the prevention and management of chronic disease in low-income and middle-income countries: a priority for primary health care. Lancet 2008;372:940-9.

15 Musinguzi G, Bastiaens $\mathrm{H}$, Wanyenze RK, et al. Capacity of health facilities to manage hypertension in Mukono and Buikwe districts in Uganda: challenges and recommendations. PLoS One 2015;10:e0142312.

16 Yan LD, Chirwa C, Chi BH, et al. Hypertension management in rura primary care facilities in Zambia: a mixed methods study. BMC Health Serv Res 2017;17:111.

17 Ministry of Health and Social Welfare (MoHSW). National noncommunicable disease strategy, 2008. Available: http://www. iccp-portal.org/sites/default/files/plans/National (TZ) NCD strategy_ 2008.pdf [Accessed 30 Aug 2020].

18 Ministry of Health, Community Development, Gender, Elderly and Children (MoHCDGEC). Strategic and action plan for the Prevention and control of non communicable diseases in Tanzania 2016 - 2020, 2016. Available: https://www.worlddiabetesfoundation.org/sites/ default/files/NCD Stategic Plan 2016 - 2020.pdf [Accessed 30 Aug 2020].

19 Mikkelsen-Lopez I, Cowley P, Kasale H, et al. Essential medicines in Tanzania: does the new delivery system improve supply and accountability? Health Syst 2014;3:74-81.

20 Shemdoe A, Mbaruku G, Dillip A, et al. Explaining retention of healthcare workers in Tanzania: moving on, coming to 'look, see and go', or stay? Hum Resour Health 2016;14:2.
21 Munga MA, Maestad O. Measuring inequalities in the distribution of health workers: the case of Tanzania. Hum Resour Health 2009;7:4.

22 World Health Organization (WHO). Primary health care systems (PRIMASYS): comprehensive case study from United Republic of Tanzania, 2017. Available: https://www.who.int/alliance-hpsr/ projects/AHPSR-PRIMASYS-Tanzania-comprehensive.pdf?ua=1 [Accessed 30 Aug 2020].

23 World Health Organization (WHO). WHO country cooperation strategy; United Republic of Tanzania; 2016-2020, 2016. Available: http://apps.who.int/iris/bitstream/10665/254890/1/ccs_tza-2016 2020_en.pdf [Accessed 30 Aug 2020].

24 Morris C, Bailey K. Measuring Health Care Quality : An Overview of Quality Measures. Issue $\mathrm{Br}$ 2014;16.

25 Islam SMS, Islam MT, Islam A, et al. National drug policy reform for noncommunicable diseases in low-resource countries: an example from Bangladesh. Bull World Health Organ 2017;95:382-4.

26 Ministry of Health and Social Welfare (MoHSW) [Tanzania Mainland], Ministry of Health (MoH) [Zanzibar], National Bureau of Statistics (NBS), Office of the Chief Government Statistician (OCGS), and ICF International 2015. Tanzania Service Provision Assessment, 2015. Available: https://dhsprogram.com/pubs/pdf/SPA22/SPA22.pdf [Accessed 30 Aug 2020].

27 Health Statistics and Information Systems, World Health Organization. Service availability and readiness assessment (SARA): an annual monitoring system for service delivery: reference manual, version 2.2, 2015. Available: http://www.who.int/healthinfo/systems/ sara_reference_manual/en/ [Accessed 30 Aug 2020]

28 Katende D, Mutungi G, Baisley K, et al. Readiness of Ugandan health services for the management of outpatients with chronic diseases. Trop Med Int Health 2015;20:1385-95.

29 Islam MR, Laskar SP, Macer D. A study on service availability and readiness assessment of non-communicable diseases using the who tool for Gazipur district in Bangladesh. Bangladesh J Bioethics 2016;7:1-13.

30 Ministry of Health and Social Welfare (MoHSW). The United Republic of Tanzania standard treatment guidelines and essential medicines list Ministry of health and social welfare. Fourth Edition, 2013. http://www.pascar.org/uploads/files/Tanzania_-_Standard_ Treatment_Guidelines_and_Essential_Medicines_List_-_Fourth_ Edition.pdf

31 Leslie HH, Spiegelman D, Zhou X, et al. Service readiness of health facilities in Bangladesh, Haiti, Kenya, Malawi, Namibia, Nepal, Rwanda, Senegal, Uganda and the United Republic of Tanzania. Bull World Health Organ 2017;95:738-48.

32 Bintabara D, Ernest A, Mpondo B. Health facility service availability and readiness to provide basic emergency obstetric and newborn care in a low-resource setting: evidence from a Tanzania national survey. BMJ Open 2019;9:bmjopen-2017-0206089.

33 Bintabara D, Nakamura K, Seino K. Determinants of facility readiness for integration of family planning with HIV testing and counseling services: evidence from the Tanzania service provision assessment survey, 2014-2015. BMC Health Serv Res 2017;17:844.

34 Peck R, Mghamba J, Vanobberghen F, et al. Preparedness of Tanzanian health facilities for outpatient primary care of hypertension and diabetes: a cross-sectional survey. Lancet Glob Health 2014;2:e285-92

35 Farzadfar F, Murray CJL, Gakidou E, et al. Effectiveness of diabetes and hypertension management by rural primary health-care workers (Behvarz workers) in Iran: a nationally representative observational study. Lancet 2012;379:47-54.

36 Checkley W, Ghannem H, Irazola V, et al. Management of NCD in low- and middle-income countries. Glob Heart 2014;9:431-43.

37 Jacobs $B$, Hill P, Bigdeli M, et al. Managing non-communicable diseases at health district level in Cambodia: a systems analysis and suggestions for improvement. BMC Health Serv Res 2016;16:32.

38 Van Minh H, Do YK, Bautista MAC, et al. Describing the primary care system capacity for the prevention and management of noncommunicable diseases in rural Vietnam. Int $J$ Health Plann Manage 2014;29:e159-73.

39 Ajuebor O, De Joncheere C, Forte G, et al. Essential medicines and basic health technologies for noncommunicable diseases : towards a set of actions to improve equitable access in Member States: WHO Discussion Paper 2015.

40 Bekele A, Getachew T, Amenu K, et al. Service availability and readiness for diabetes care at health facilities in Ethiopia. Ethiop $J$ Heal Dev 2017:31:110-9.

41 Oyekale AS. Assessment of primary health care facilities' service readiness in Nigeria. BMC Health Serv Res 2017;17:172.

42 Jiwa M, Millett S, Meng X, et al. Impact of the presence of medical equipment in images on viewers' perceptions of the trustworthiness of an individual on-screen. J Med Internet Res 2012;14:e100. 
43 Kearney PM, Whelton M, Reynolds K, et al. Global burden of hypertension: analysis of worldwide data. Lancet 2005;365:217-23.

44 Akinlua JT, Meakin R, Umar AM, et al. Current prevalence pattern of hypertension in Nigeria: a systematic review. PLoS One 2015;10:e0140021.

45 Kibret KT, Mesfin YM. Prevalence of hypertension in Ethiopia: a systematic meta-analysis. Public Health Rev 2015;36:14.

46 Ait-Khaled N, Enarson D, Bousquet J. Chronic respiratory diseases in developing countries: the burden and strategies for prevention and management. Bull World Health Organ 2001;79:971-9.

47 Jaffar HY, Alhaid TA, Al-Hassan NA, et al. Knowledge, attitude and practice of physicians toward peak expiratory flow meter in primary health care centers in Kuwait. Alexandria J Med 2011;47:165-71.

48 Hajia AM, Mohammed FAK, Al-Saqer MA, et al. Knowledge, attitude and practice of nurses toward peak expiratory flow meter in primary health care centers in Kuwait. Alexandria J Med 2011;47:255-60.

49 World Health Organization (WHO). Prevention of recurrent heart attacks and strokes in low and middle income populations: evidencebased recommendations for policy makers and health professionals, 2013. Available: http://www.who.int/cardiovascular_diseases/ resources/pub0402/en/ [Accessed 30 Aug 2020].

50 Bollyky TJ. Access to drugs for treatment of noncommunicable diseases. PLoS Med 2013;10:e1001485.

51 Cameron A, Roubos I, Ewen M, et al. Differences in the availability of medicines for chronic and acute conditions in the public and private sectors of developing countries. Bull World Health Organ 2011;89:412-21.

52 Robertson J, Macé C, Forte G, et al. Medicines availability for non-communicable diseases: the case for standardized monitoring. Global Health 2015;11:18.

$53 \mathrm{WHO} / \mathrm{HAl}$. Measuring medicine prices, availability, affordability and price components. 2nd Edition. Geneva, Switzerland, 2008. http:// apps.who.int/iris/handle/10665/70013

54 Volman B, Leufkens B, Stolk P, et al. Direct costs and availability of diabetes medicines in low-income and middle-income countries, 2008. Available: http://digicollection.org/hss/documents/s18387en/ s18387en.pdf
55 Mendis S, Fukino K, Cameron A, et al. The availability and affordability of selected essential medicines for chronic diseases in six low- and middle-income countries. Bull World Health Organ 2007;85:279-88.

56 Whitworth JA, World Health Organization, International Society of Hypertension Writing Group. 2003 World Health Organization (WHO)/International Society of Hypertension (ISH) statement on management of hypertension. J Hypertens 2003;21:1983-92.

57 Jingi AM, Noubiap JJN, Ewane Onana A, et al. Access to diagnostic tests and essential medicines for cardiovascular diseases and diabetes care: cost, availability and affordability in the West region of Cameroon. PLoS One 2014;9:e111812.

58 World Health Organization (WHO). Implementation tools package of essential noncommunicable (PEN) disease interventions for primary health care in low-resource settings. Available: http://apps.who.int/ iris/bitstream/10665/133525/1/9789241506557_eng.pdf [Accessed 30 Aug 2020].

59 Cameron A, Ewen M, Ross-Degnan D, et al. Medicine prices, availability, and affordability in 36 developing and middle-income countries: a secondary analysis. Lancet 2009;373:240-9.

60 Ewen M, Zweekhorst M, Regeer B, et al. Baseline assessment of WHO's target for both availability and affordability of essential medicines to treat non-communicable diseases. PLoS One 2017:12:e0171284

61 Bissell K, Perrin C, Beran D. Access to essential medicines to treat chronic respiratory disease in low-income countries. Int J Tuberc Lung Dis 2016;20:717-28.

62 Bazargani YT, de Boer A, Leufkens HGM, et al. Essential medicines for COPD and asthma in low and middle-income countries. Thorax 2014:69:1149-51.

63 Sikika. Medicines and medical supplies availability report. using absorbent gauze availability survey as an entry point. A case of 71 districts and 30 health facilities across mainland Tanzania, 2011. Available: http://sikika.or.tz/wp-content/uploads/2013/09/medicinesand-medical-and-supply-availability-report.pdf [Accessed 30 Aug 2020].

64 Ministry of Health and Social Welfare (MoHSW). In-Depth assessment of the medicines supply system in Tanzania, 2008. Available: http://apps.who.int/medicinedocs/documents/s16503e/ s16503e.pdf [Accessed 30 Aug 2020]. 